\title{
Efecto del tiempo y temperatura en la deshidratación de oca (Oxalis Tuberosa Mol.) Mediante lecho fluidizado para la obtención de harina
}

\author{
Effect of time and temperature on the dehydration of oca (Oxalis tuberosa Mol.) Through \\ fluidized bed for the obtaining of flour \\ "Efeito do tempo e da temperatura na desidratação de oca (oxalis tuberosa mol.) Através \\ de cama fluidizada para obter farinha"
}

\author{
- Franklin Ore Areche \\ frankin.ore@unh.edu.pe \\ Código ORCID: 0000-0002-7168-1742
}

\author{
- Lissete Lourdes Aguirre Huayhua \\ lissete.aguirre@unh.edu.pe \\ Código ORCID: 0000-0003-2450-5153
}

\section{- Jovencio Ticsihua Huaman \\ jovencio.ticsihua@unh.edu.pe \\ Código ORCID: 0000-0001-5287-4461}

Universidad Nacional de Huancavelica - Escuela Profesional de Ingeniería agroindustrial Huancavelica - Perú

Artículo recibido 23 de octubre 2020 / Arbitrado y aceptado 21 de noviembre 2020 / publicado 18 de diciembre 2020

\section{RESUMEN}

La región andina es conocida por ser cuna de algunos cultivos andinos de importancia mundial como la papa, oca, olluco y mashua. La oca es un tubérculo muy extendido en los Andes, por ser un producto tradicional, ancestral de sabor agradable, es uno de los alimentos más apreciados en la alimentación del sector rural. La Oxalis tuberosa Mol. Representa una buena fuente de antioxidantes por lo que puede considerarse como una excelente opción de materia prima para la elaboración de alimentos funcionales. Los objetivos de la investigación realizada fueron: evaluar las propiedades fisicoquímicas de la harina de oca, evaluar las propiedades químico proximal de la harina de oca y realizar el análisis microbiológico de la harina de oca (variedad rosada) deshidratado en lecho fluidizado a diferentes temperaturas $\left(60,70\right.$ y $\left.80^{\circ} \mathrm{C}\right)$ por un tiempo de 60 minutos a cada uno. Se empezó obteniendo las harinas, paro lo cual se seleccionó, soleo, limpió, lavo, cortó, escaldo, deshidrato, enfrió, molió, tamizo y envaso. En la caracterización fisicoquímica se hizo el análisis proximal, se determinó el $\mathrm{pH}$ y acidez mediante valoración volumétrica, se utilizó el método refractométrico para medir la concentración de azúcar, para el análisis microbiológico, la muestra se obtuvo al azar. Los resultados del análisis químico proximal realizados fueron: humedad, grasa, proteína, fibra, ceniza y carbohidratos, los cuales no muestran diferencias significativas entre ellos, los resultados microbiológicos indican que la harina de oca se encuentra dentro de los parámetros microbiológicos dados por DIGESA (2000), Se puede destacar que la harina de oca mantiene las características organolépticas propias del producto para su uso agroindustrial.

\begin{abstract}
The Andean region is known for being the cradle of some Andean crops of world importance such as potatoes, oca, olluco and mashua. The goose is a very widespread tuber in the Andes, as it is a traditional, ancestral product with a pleasant taste, it is one of the most appreciated foods in the rural sector. The Oxalis tuberosa Mol. represents a good source of antioxidants so it can be considered as an excellent option of raw material for the elaboration of functional foods. The objectives of the research carried out were: to evaluate the physicochemical properties of the goose meal, to evaluate the proximal chemical properties of the goose meal and to perform the microbiological analysis of the goose meal (pink variety) dehydrated in a fluidized bed at different temperatures $\left(60,70\right.$ and $\left.80^{\circ} \mathrm{C}\right)$ for a time of 60 minutes each. It began by obtaining the flours, for which it was selected, soleus, cleaned, washed, cut, blanched, dehydrated, cooled, ground, sieved and packed. In the physicochemical characterization, the proximal analysis was made, the $\mathrm{pH}$ and acidity were determined by volumetric titration, the refractometric method was used to measure the sugar concentration, for the microbiological analysis, the sample was obtained at random. The results of the proximal chemical analysis performed were: moisture, fat, protein, fiber, ash and carbohydrates, which do not show significant differences between them, the microbiological results indicate that the goose flour is within the microbiological parameters given by DIGESA (2000). It can be noted that goose flour maintains the organoleptic characteristics of the product for its agroindustrial use.
\end{abstract}

Key words: Fluidized bed; flour; Oxalis tubosa, dehydration

Palabras clave: Lecho fluidizado; harina; Oxalis tuberosa, deshidratación 


\section{RESUMO}

A região andina é conhecida por ser o berço de algumas culturas andinas de importância mundial, como a batata, a oca, o olluco e o mashua. 0 ganso é um tubérculo muito difundido na Cordilheira dos Andes, pois é um produto tradicional, ancestral e de sabor agradável, é um dos alimentos mais apreciados no setor rural. The Oxalis tuberosa Mol. representa uma boa fonte de antioxidantes, podendo ser considerada uma excelente opção de matéria-prima para a elaboração de alimentos funcionais. Os objetivos da pesquisa realizada foram: avaliar as propriedades físico-químicas da farinha de ganso, avaliar as propriedades químicas proximais da farinha de ganso e realizar a análise microbiológica da farinha de ganso desidratada (variedade rosa) em leito fluidizado a diferentes temperaturas $\left(60,70\right.$ e $\left.80^{\circ} \mathrm{C}\right)$ por um tempo de 60 minutos cada. Iniciou-se pela obtenção das farinhas, para as quais foi selecionado, sóleo, limpado, lavado, cortado, branqueado, desidratado, resfriado, moído, peneirado e embalado. $\mathrm{Na}$ caracterização físico-química, foi feita a análise proximal, o pH e a acidez foram determinados por titulação volumétrica, o método refratométrico foi utilizado para medir a concentração de açúcares, para a análise microbiológica, a amostra foi obtida de forma aleatória. Os resultados da análise química proximal realizada foram: umidade, gordura, proteína, fibra, cinzas e carboidratos, os quais não apresentam diferenças significativas entre si, os resultados microbiológicos indicam que a farinha de ganso está dentro dos parâmetros microbiológicos dados por DIGESA (2000). Nota-se que a farinha de ganso mantém as características organolépticas do produto para seu uso agroindustrial.

Palavras-chave: Leito fluidizado, farinha, Oxalis tubosa, desidratação

\section{INTRODUCCIÓN}

La región andina es conocida por ser cuna de algunos cultivos de importancia mundial como la papa, el camote y los ajíes. Sin embargo, gran parte de las plantas alimenticias domesticadas por los pueblos andinos son poco conocidas internacionalmente, incluso en los mismos países andinos. Entre estas plantas se encuentran las raíces y los tubérculos andinos, un grupo de nueve especies que incluye: achira, ahipa, arracacha, maca mauka, oca, ulluco y yacón. Todos ellos son importantes en la alimentación del poblador andino rural, pero su aprovechamiento está restringido (1).

En la actualidad como cambio en el estilo de vida, ha influido de manera importante sobre los hábitos de alimentación, el consumo de alimentos procesados está aumentando a un ritmo acelerado (2), sin embargo, los consumidores están tomando conciencia de la importancia de la dieta en relación con los problemas de salud (3); de ahí el interés por conocer más acerca de los alimentos que tienen un impacto en la salud, ha crecido de manera considerable; Es por ello que el desarrollo de alimentos, a base de materias primas no convencionales, que aporten compuestos bioactivos, ha tenido gran interés en los últimos años. Este tipo de alimentos son llamados comúnmente alimentos funcionales. Alimento funcional se define como aquel que tiene uno o más componentes específicos, que tienen influencia positiva en la salud y el bienestar del consumidor (4).

La mayoría de los antioxidantes se encuentra en alimentos vegetales, y en los cultivos andinos, ricos en compuestos fenólicos que son considerados metabolitos secundarios de las plantas, existe evidencia creciente que demuestra el efecto benéfico de los mismos en la salud y nutrición humana, este se basa en la capacidad antioxidante y sus posibles beneficios a la salud tales como reducción de enfermedades coronarias, mejora de la agudeza visual, y actividades anti cancerígenas (5). Existen nuevas fuentes botánicas, las cuales se busca que sean explotadas por su alto contenido en compuestos bioactivos los cuales dan grandes beneficios a la salud; una de esas nuevas fuentes es la Oxalis tuberosa, que constituye una fuente de recursos poco conocidos y explotados que representan posibilidades para la agricultura, la alimentación, la agroindustria y el comercio internacional.

La Oxalis tuberosa Mol. representa una buena fuente de antioxidantes por lo que puede considerarse como una excelente opción de materia prima para la elaboración de alimentos con propiedades funcionales (6). Para la FAO (7) la Oxalis tuberosa es una planta herbácea de consistencia no leñosa, que vive más de dos años. Una vez cultivada en un lugar, si las condiciones son las adecuadas, pueden crecer y 
florecer por muchos años sin demasiadas exigencias, tiene un tallo erguido en la primera fase de desarrollo que pasa a ceder más adelante, a medida que madura. "La Oxalis tuberosa es una de las plantas que almacena más almidón durante el invierno época en la que no está creciendo. Los tubérculos tienen forma elíptica o cilíndrica y pueden ser de color blanco, amarillo, rojo y púrpura. Su longitud varía entre 5 y $7.5 \mathrm{~cm}$ y su diámetro, entre 2.5 y $3.75 \mathrm{~cm}$.

La oca (Oxalis tuberosa), al igual que otros tubérculos andinos como la papa, papa lisa o el isaño, es un tubérculo originario de la región andina de América, y se produce principalmente entre Perú, Bolivia y Ecuador. En orden de importancia alimenticia y económica, ocupa el segundo lugar después de la papa Emshwiller (8). Según National Research Council (9), los tubérculos de oca tienen una alta variación en sus niveles nutritivos. Como promedio posee un $84.1 \%$ de agua, $1.1 \%$ de proteína, $13.2 \%$ de carbohidratos, $0.6 \%$ grasa y $1 \%$ de fibra. El contenido vitamínico varía, pero puede tener cantidades significativas de retinol (vitamina A) y los tubérculos amargos contienen hasta 500 ppm de ácido oxálico. En este sentido, Campos et al., (10) señala al tubérculo de oca como una excelente fuente alimenticia y nutricional en la dieta alimenticia del poblador peruano.

El secado o deshidratado es una de las tecnologías más frecuentes en la agroindustria y consiste en la eliminación de gran parte del agua del producto procesado, la evaporación del agua se hace a través de una corriente de aire caliente, la cual transmite el calor latente de evaporación al producto. Lo que se busca es disminuir al máximo la actividad bioquímica interna y la acción de microorganismos que permitan mantener por mucho más tiempo el producto en condiciones de almacenaje (11). La deshidratación o secado se realiza para aumentar la vida útil de los alimentos, para disminuir los costos de transporte, empaque y de almacenamiento, para suplir las necesidades de materias primas secas como ingredientes para otros productos, así como en el desarrollo de nuevos productos. El proceso de deshidratación generalmente se realiza por medio de un secado térmico utilizando técnicas como secado con aire, al sol y a vacío, microondas y liofilización, pero con la consecuente modificación de las propiedades organolépticas.

Una alternativa más comúnmente utilizada en la industria de alimentos para obtener productos deshidratados, es el secado convectivo, pues ofrece un proceso relativamente rápido, higiénico, uniforme, (12) $\mathrm{y}$ disminuye gastos de embalaje, almacenamiento y transporte, Ateeque et al., (13). Sin embargo, el elevado costo de los combustibles y la ineficiencia de algunos métodos de secado, ha conducido a utilizar procesos alternativos que permitan alcanzar las mejores condiciones en el producto, (14). Los secadores de lecho fluidizado son algunos de los sistemas más utilizados en la industria, debido a que presentan ventajas en comparación con secadores de bandejas o de vacío rotativo, Srinivas et al., (15), en ellos se imprimen velocidad suficiente a un lecho de partículas para dispersarlas y generar turbulencia que acelera la deshidratación, Sánchez-Ramírez et al., (16). El lecho fluidizado ofrece excelentes tasas de transferencia de calor y masa gracias a su alto grado de mezcla, al igual que una distribución uniforme de la temperatura, $(17,18)$ debido a que se genera un área superficial de contacto muy alta entre el gas y el sólido presente en el lecho, (15), dando como resultado una reducción en el tiempo de proceso, (16). 


\section{MATERIALES Y MÉTODOS}

\section{Análisis fisicoquímico de la harina de oca}

\section{Sólidos solubles (Brix)}

Se determinó el contenido de sólidos solubles por medio del método de la AOAC 932.12/80 de la Association of Official Analytical Chemists - AOAC Internacional (19). Los grados Brix, sirven para determinar el cociente total de sacarosa disuelta en un líquido, es la concentración de sólidos solubles, la lectura se realizó por medio de un refractómetro.

\section{pH (método electrométrico)}

Recomendado por la AOAC 981.12 de la Association of Official MAnalytical Chemists AOAC Internacional (19). Mediante un potenciómetro digital a $20{ }^{\circ} \mathrm{C}$, la medición se realizó en una solución filtrada de $10 \mathrm{~g}$ del tubérculo en $100 \mathrm{ml}$ de agua destilada.

\section{\% Acidez Total}

Método recomendado por la AOAC 939.05 (2016) (19) de la Association of Official Analytical Chemists - AOAC Internacional. En alimentos el grado de acidez indica el contenido en ácidos libres. Se determinó mediante una valoración (volumetría) con un reactivo básico. El resultado se expresó como el porcentaje (\%) del ácido predominante en el tubérculo, para determinar el \% de acidez se tiene en cuenta la siguiente ecuación.

$$
\begin{gathered}
\% \text { de acides }\left(\frac{\mathrm{g}}{100 \mathrm{ml}}\right)= \\
\left.\frac{\text { Gasto } x(\mathrm{NaOH}) \times \text { pmeq } \times \mathrm{fv}}{T M(\mathrm{ml})} \times 100 \text { (Ec. } 1\right)
\end{gathered}
$$

\section{Donde}

Gasto: ml gastados del titulante $(\mathrm{NaOH})$

NaOH: Normalidad del titulante

pmeq: Peso miliequivalente del ácido predomínate en la muestra

fv: Factor de valoración del titulante $(0,045$ ácido oxálico)

TM: Tamaño o peso de la muestra en (ml).

\section{Esquema del secador de Lecho fluidizado}

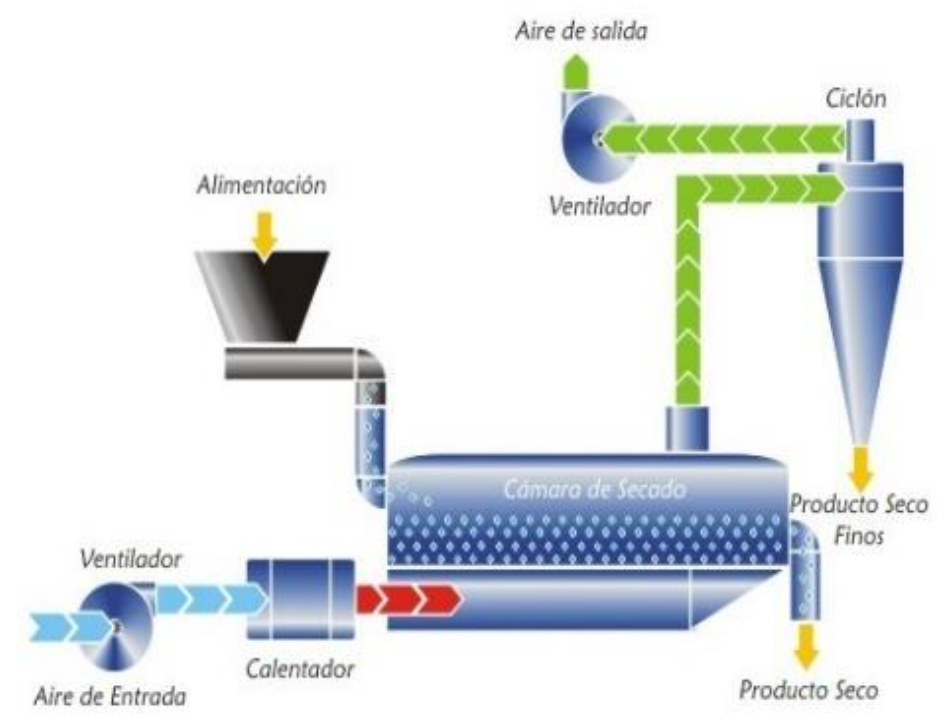

Figura 1. Vista esquemática de un secador de lecho fluidizado (ciclo abierto) 


\section{Obtención de la harina de oca}

Los tubérculos de oca fueron recolectados del distrito y provincia de Acobamba Huancavelica, las cuales se seleccionaron cuidadosamente, por tamaño $(2.5 \mathrm{~cm}$ de diámetro por $8.0 \mathrm{~cm}$ de largo aproximadamente) y la variedad fue la rosada.

Para la obtención de la harina de oca deshidratada mediante lecho fluidizado se siguió el siguiente diagrama.

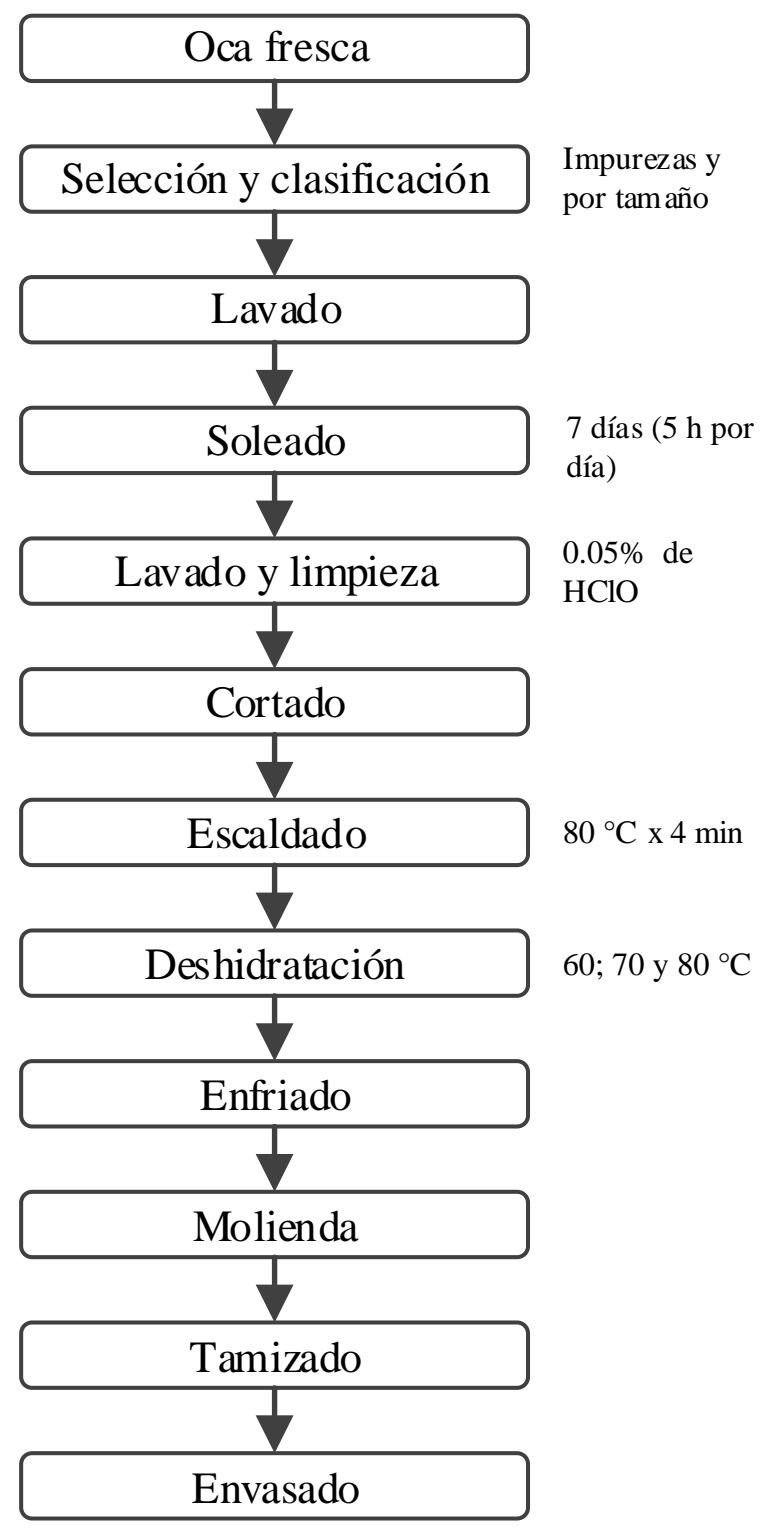

Figura 2. Diagrama de flujo para la obtención harina de oca deshidratada mediante lecho fluidizado. 


\section{Descripción del proceso de obtención de} harina de oca

\section{Selección y clasificación}

Se escogió la oca fresca, sana que no presentaba ningún daño mecánico, ni principios de descomposición por efectos microbianos. Se clasificaron los tubérculos de tamaño mediano (2.5 $\mathrm{cm}$ de diámetro por $8.0 \mathrm{~cm}$ de largo) aproximadamente.

\section{Lavado}

Se hizo de forma rápida con agua potable, con la finalidad de eliminar los restos de tierra. Se realizó por inmersión.

\section{Soleado}

Se expuso al sol durante 7 días, desde las 9 am hasta las $2 \mathrm{pm}$, con la finalidad de reducir el agua presente en el tubérculo, además de disminuir el ácido oxálico e incrementar la concentración de azúcar.

\section{Lavado y limpieza}

Se hizo de forma rápida con agua fría y desinfectante, con la finalidad de eliminar la carga microbiana. Esta operación se realizó por inmersión. Las soluciones desinfectantes, a emplear, estuvieron compuestas de hipoclorito de sodio (lejía) en una concentración $0.05 \%$.

\section{Cortado}

Se hizo cortes transversales con la ayuda de un cuchillo, se cortaron en trozos de $1 \mathrm{~cm}$ de espesor. Para que las láminas de rodajas tengan un secado uniforme.

\section{Escaldado}

El escaldado se realizó para inhibir el efecto producido por la acción enzimática, este proceso se realizó durante $4 \mathrm{~min}$ en agua a punto de ebullición a $80{ }^{\circ} \mathrm{C}(20,21)$. Esto con el fin de inhibir la acción de las enzimas que provocan el pardeamiento (22). Transcurrido el tiempo, inmediatamente se realizó un choque térmico con agua fría a $6^{\circ} \mathrm{C}$.

\section{Deshidratación}

Se colocaron $1500 \mathrm{~g}$ de oca cortada y escaldada en el secador de lecho fluidizado, donde se utilizó tres temperaturas $(60 ; 70 \mathrm{y}$ $80^{\circ} \mathrm{C}$ ).

\section{Enfriado}

Se llevó a cabo por espacio de 5 minutos

\section{Molienda}

Al producto deshidratado se le disminuyo el tamaño de partícula por medio de un molino de martillo de marca Vulcano.

\section{Tamizado}

Se realizó con malla $\mathrm{N}^{\circ} 100$ para obtener una harina con granulometría fina.

\section{Envasado}

Las muestras de harina obtenida se pesaron en fracciones de $1 / 4$ de $\mathrm{kg}$ y se envasaron en bolsas de polietileno, selladas térmicamente.

\section{Análisis microbiológico}

Se determinó el recuento de Aerobios Mesófilos viables (UFC/g), Numeración de hongos y levaduras (UFC/g) y Numeración de Coliformes (UFC/g), para ello se pesaron asépticamente muestras de $10 \mathrm{~g}$ de harina de oca obtenida a diferentes temperaturas y se mescló con $90 \mathrm{ml}$ de agua peptonada estéril al = $1 \%$ por 3 minutos a temperatura ambiente. Se preparó diluciones decimales en serie tomando $1 \mathrm{ml}$ de la solución anterior en $9 \mathrm{ml}$ de agua peptonada por duplicado, se vertieron y se extendieron para el recuento total en Plate 
Count Agar luego de incubar a $30{ }^{\circ} \mathrm{C}$ por 48 horas.

\section{Análisis estadístico}

Se utilizó el programa IBM SPSS Statistics

25. Los datos obtenidos mediante la prueba hedónica fueron transformados a valores numéricos para determinar la media. Se realizó un análisis de varianza para determinar las diferencias entre fórmulas y atributos.

\section{RESULTADOS Y DISCUSIÓN}

\section{Análisis fisicoquímico de la harina de oca}

Tabla 1. Resultados del análisis fisicoquímico de la harina de oca deshidratado a 3 temperaturas.

\begin{tabular}{cccc}
\hline \multirow{2}{*}{ Análisis } & \multicolumn{3}{c}{ Resultados } \\
\cline { 2 - 4 } & $\mathbf{6 0}^{\circ} \mathbf{C}$ & $\mathbf{7 0}^{\circ} \mathbf{C}$ & $\mathbf{8 0}{ }^{\circ} \mathbf{C}$ \\
\hline Brix & 22.00 & 22.10 & 22.10 \\
pH & 5,55 & 5,50 & 5,50 \\
Acidez & 0.18 & 0.17 & 0.17
\end{tabular}

En la tabla 1 se muestra los resultados del análisis fisicoquímico de la harina de oca deshidratado en lecho fluidizado a 3 diferentes temperaturas, donde se puede observar que el contenido de sólidos solubles para la harina de oca es de 22.10 a 20.10 oBrix, donde no existe diferencia significativa, es decir los sólidos solubles de la harina no son diferentes, además Palate (23) menciona que la disminución de la acidez se relaciona con el aumento de sólidos solubles, es así que la harina de oca presenta menor acidez, pero contiene mayor sólidos solubles, esto permitirá un menor consumo de azúcar (sacarosa) en la formulación de productos como indica Caiza (24), asimismo un índice superior de refracción tendrán mayor contenido de azúcar, mayor contenido de proteínas y mayor densidad, es así que esto se puede evidenciar con los resultados reportados de la harina de oca presentando mayor porcentaje en proteínas y mayor densidad; mientras que donde la harina de oca presenta pH entre 5.69 a 5.50 cercano a la oca deshidrata 6.3 según indica Cajamarca (25) siendo ligeramente ácido, lo cual hace estar propensa a desarrollo microbiano, sin embargo, la harina de oca presenta un $\mathrm{pH}$ menor de 5.60 en consecuencia, es menos propensa al desarrollo microbiano.

Respecto a la acidez Egan, Kirk y Sawyer (26) indican que la acidez de las harinas no debe exceder el $0.2 \%$, como se puede ver las tres harinas están dentro de este rango, esto demuestra que presentan un buen estado de conservación, sin embargo, existe diferencias significativas entre ambas harinas, el porcentaje de acidez es diferente. 


\section{Análisis químico proximal de la oca fresca y harina de oca}

Tabla 2. Resultados del análisis químico proximal de la harina de oca deshidratado a tres temperaturas.

\begin{tabular}{ccccc}
\hline \multirow{2}{*}{ Análisis } & \multicolumn{5}{c}{ Resultados } \\
\cline { 2 - 5 } & Oca fresca & $\mathbf{6 0}^{\circ} \mathbf{C}$ & $\mathbf{7 0}^{\circ} \mathbf{C}$ & $\mathbf{8 0}{ }^{\circ} \mathbf{C}$ \\
\hline Humedad (\%) & 81.92 & 11.32 & 9.30 & 9.00 \\
Grasa (\%) & 0.09 & 0.66 & 0.54 & 0.52 \\
Proteína (\%) & 1.43 & 3.74 & 3.30 & 3.30 \\
Fibra (\%) & 1.23 & 5.96 & 2.97 & 2.96 \\
Ceniza (\%) & 2.01 & 4.39 & 3.49 & 3.35 \\
Carbohidratos & 13.32 & 75.93 & 80.40 & 80.87 \\
\hline
\end{tabular}

En la tabla 2 se muestra los resultados obtenidos del análisis fisicoquímico de la oca fresca y de la harina de oca deshidratada a $60^{\circ} \mathrm{C}, 70^{\circ} \mathrm{C}$ y $80^{\circ} \mathrm{C}$, todos deshidratados por 60 minutos. Se puede apreciar que los resultados para la oca fresca son aproximados a los de Collazos (27), estas ligeras variaciones pueden ser por la procedencia de la oca fresca o el tiempo de cosecha; mientras que los resultados de la harina de oca están dentro de los rangos de humedad para las harinas (13-15), como menciona la NTP (28), y los demás valores están próximo a lo reportado por Cajamarca (25).

\section{Resultados microbiológicos}

Tabla 3. Resultados del análisis microbiológico de la harina de oca deshidratado a tres temperaturas.

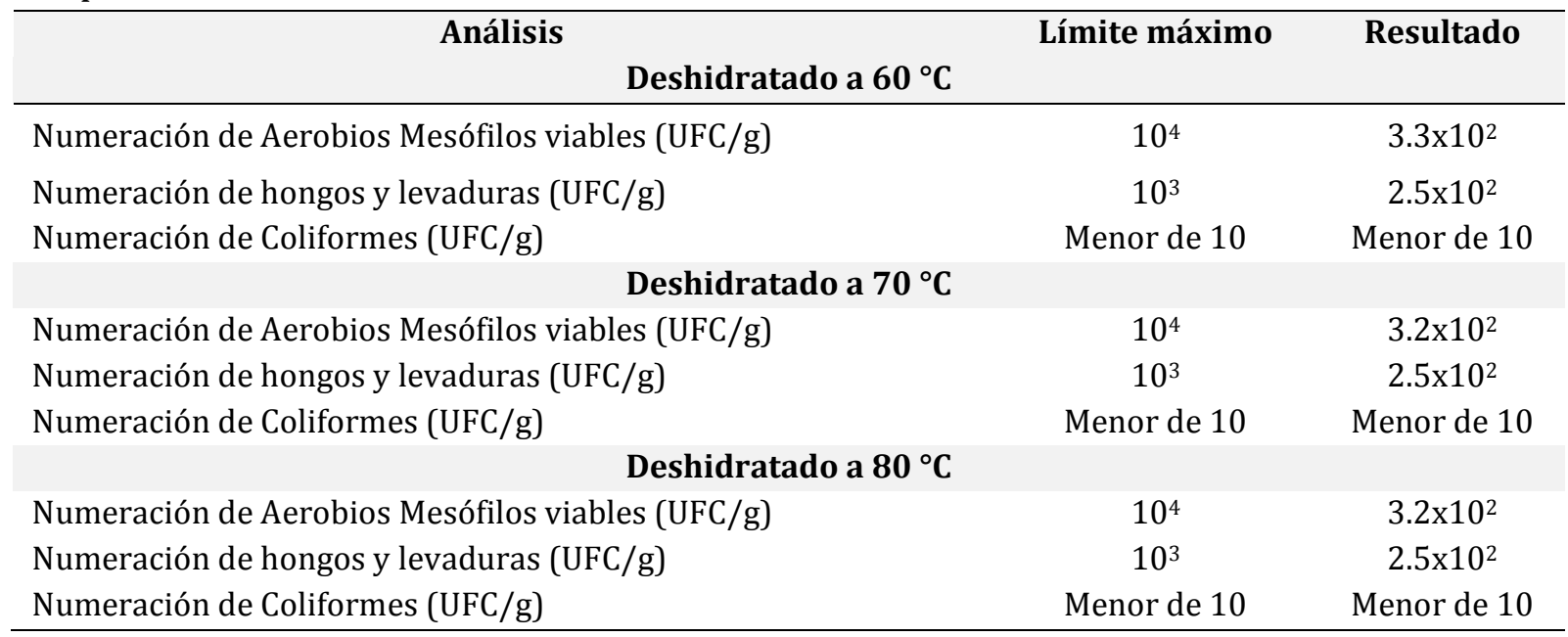


La harina de oca fue evaluada después de 40 días de almacenamiento con los siguientes resultados: Numeración de Aerobios Mesófilos viables (UFC/g) de 3.3×102; Numeración de hongos y levaduras (UFC/g) de 2.5x102 y Numeración de Coliformes (UFC/g) reportando ausencia. Los resultados indican que la harina de oca se encuentra dentro de los parámetros de los requisitos microbiológicos dadas por DIGESA (29), donde señala que toda harina debe encontrarse exenta de microorganismos patógenos, los rangos permisibles para el caso de las harinas son las siguientes: Recuento de Mesófilos viables de 102 a 106 hongos y levaduras de 102 a 104 y Coliformes totales menor a 10 y por lo tanto la harina de se encuentra en condiciones óptimas para su consumo y comercialización.

\section{CONCLUSIONES}

Se obtuvo harina de oca (Oxalis tuberosa Mol.) por deshidratación con lecho fluidizado a 3 diferentes temperaturas, donde se determinó la propiedad fisicoquímica de las muestras obtenidas, con respecto a la acidez se observó que no se encontraron diferencias significativas en las muestras obtenidas.

Se evaluó las propiedades químico proximal de las harinas obtenidas a diferentes temperaturas, donde se puede observar que la harina obtenida a $60{ }^{\circ} \mathrm{C}$ es la que tiene mayores porcentajes y en carbohidratos es inferior a comparación de los deshidratados a temperaturas de $70^{\circ} \mathrm{C}$ y $80^{\circ} \mathrm{C}$.

Se realizó el análisis microbiológico de las harinas de oca obtenidas a diferentes temperaturas, donde se pudo observar una ligera diferencia en la numeración de aerobios mesófilos viables en la muestra obtenida a $60^{\circ} \mathrm{C}$.

\section{REFERENCIAS BIBLIOGRÁFICAS}

1. Maldonado, S., Santapaola, J., Singh, J., Torrez, M., y Garay, A. (2008). Cinética de la transferencia de masa durante la deshidratación osmótica de yacón (Smallanthus sonchifolius). Food Science and Technology, 28(1), 251-256. https://doi.org/10.1590/S010120612008000100036

2. Mohammed, S. (2016). Optimization of the extrusion process for development of high fibre soybean-rice ready-to-eat snacks using carrot pomace and cauliflower trimmings. Food Science and Technology, 135-144

3. Arribas, C., Cabellos, B., Cuadrado, C., Guillamón, E., y Pedrosa, M. (2017). The impact of extrusion on the nutritional composition, dietary fiber and in vitro digestibility of gluten-free snacks based on rice, pea and carob flour blends. Food and function, 3654-3663

4. Sarmiento, L. (2006). Alimentos funcionales, una nueva alternativa de alimentación. ORINOQUIA, 16-23

5. Robles, N. (2016). Efecto del tiempo y temperatura de pasteurización en el contenido de vitamina c y capacidad antioxidante en zumo de oca (Oxalis tuberosa Mol). Tesis para optar el título profesional. Universidad Nacional del Altiplano. Puno - Perú. Disponible en: http://181.176.163.136/bitstream/handl e/UNAP/3592/Robles_Condori_Nelly.pdf ?sequence $=1$ yisAllowed $=y$

6. Chirinos, R., Betalleluz-Pallardel, I., Huamán, A., Arbizu, C., Pedreschi, R., y Campos, D. (2009). HPLC-DAD characterisation of phenolic compounds from Andean oca (Oxalis tuberosa Mol.) tubers and their contribution to the antioxidant capacity. Food Chemistry, $1243-1251$ 
7. FAO (2018). Oxalis tuberosa Molina. Organización de las Naciones Unidas para la Agricultura y la Alimentación. Cultivo tradicional del mes. Recuperado de: http://www.fao.org/traditionalcrops/oca/es/

8. Emshwiller, E. (2002). Biogeography of theOxalis tuberosa alliance. The Botanical Review, 68(1), 128.

9. National Research Council. (1989). Lost crops of the Incas: little-known plants of the Andes with promise for worldwide cultivation: National Academies Press.

10. Campos, D., Noratto, G., Chirinos, R., Arbizu, C. y Roca, W. (2006). Antioxidant capacity and secondary metabolites in four species of Andean tuber crops: native potato (Solanum sp), mashua (Tropaelum tuberosum Ruiz y Pavon), Oca (Oxalis tuberosa M.) and Olluco (Ullucus tuberosus Caldas). Journal of Science Food and Agriculture 86, 14811488

11. Romero, C. (2000). Deshidratación Osmótica de Tomate de Árbol (Cyphomandrabetacea). Journal, FoodSciences: Estados Unidos

12. Vásquez-Parra, J., Ochoa-Martínez C. y. Bustos-Parra, M. (2013). Effect of Chemical and Physical Pretreatments on the Convective Drying of Cape Gooseberry Fruits (Physalis peruviana), doi: 10.1016/j.jfoodeng.2013.06.037, J. Food Eng, 119(3), 648-654

13. Ateeque, M., Mishra, R., Chandramohan, $P$. y Talukdar, P. (2014). Numerical Modeling of Convective Drying of Food with Spatially Dependent Transfer Coefficient in a Turbulent Flow Field, doi: 10.1016/j.ijthermalsci.2013.12.003, Int. J. Therm. Sci, 78, 145-157

14. Ocampo, A. (2006). Modelo Cinético del Secado de la Pulpa de Mango, ISSN: 17941237, Revista EIA, 3(5), 119-128

15. Srinivas, G., Thamida S. y. Setty, Y. (2015). Simulation and validation of a model for a batch wall heated fluidized bed dryer, doi: $\quad$ 10.1016/j.powtec.2014.10.011, Powder Technol, 270, 368-377
16. Sánchez-Ramírez, J., Anaya-Sosa, I. (2007). Estudio de la Hidrodinámica del Café Tostado (Coffea arabica L.) en Lecho Fluidizado, ISSN: 1665-2738, Rev. Mex. Ing. Quim., 6(2), 185-192

17. Torrez Irigoyen, R. y Giner, S. (2016). Drying-Toasting of Presoaked Soybean in Fluidised Bed. Modeling, Validation and Simulation of Operational Variants for Reducing Energy Consumption, doi: 10.1016/j.jfoodeng.2015.10.011, Journal of Food Engineering, 171, 78-86

18. $\mathrm{Xu}, \mathrm{SY}$, Belopolski, I., Alidoust, N., Neupane, M., Bian, G., Zhang, C., y Hasan, MZ. Descubrimiento de un semimetal de Weyl fermion y arcos de Fermi topológicos. Science, (2015)349 (6248), 613-617

19. AOAC 934.06, (2016). Official Methods of Analysis, Moisture in Dried Fruits, 2940, Gaithersburg, Estados Unidos

20. Fonseca K., y Romero J., (2012) Evaluación de harinas precocidas y no precocidas de clones promisorios de papa criolla Solanum tuberosum Grupo phureja para aprovechamiento industrial. Trabajo de grado no publicado. Universidad de la Salle. Bogotá

21. Ramallo R. (2004). Análisis exploratorio de los ácidos grasos del Isaño (Tropaeolum tuberosum). UPB Investigación y Desarrollo. No 4 pág. (7172)

22. Beltrán, A. y Mera, J. (2014). Elaboración del tubérculo Mashua (Tropaeolum tuberosum) troceada en miel $y$ determinación de capacidad antioxidante. Tesis de grado, Universidad de Guayaquil.

23. Palate, A. (2013). Estudio del efecto de la temperatura y el tiempo en las características físico-químicas y sensoriales de la oca (Oxalis tuberosa) durante su maduración. Ecuador-Ambato

24. Caiza, C. (2010). Elaboración y valoración nutricional de tres productos alternativos a base de oca (Oxalis tuberosa) para escolares del proyecto runa kawsay. Tesis de grado. Ecuador 
25. Cajamarca, E. (2010). Evaluación nutricional de la Oca (Oxalis tuberosa) fresca; endulzada y deshidratada en secador de bandejas. Tesis de grado, Facultad de Ingeniería Químico Farmacia, Escuela Politéctnica Chimborazo

26. Egan H. Kirk, R. y Sawyer, R. (1981). Análisis químico de alimentos. Editorial C.E.C.S.A. México. 28

27 Collazos, C. (1990). Tabla de Composición de los Alimentos Peruanos. Instituto Nacional de Nutrición. Lima - Perú
28. Programa Nacional de Toxicología (NTP). (1986). Estudios de toxicología y carcinogénesis del NTP del óxido de decabromodifenilo (número CAS 116319-5) en ratas F344 / N y ratones B6C3F1 (estudios de alimentación). Serie de informes técnicos del Programa Nacional de Toxicología, 309, 1-242

29. DIGESA. (2000). Evaluación de la Calidad del Aire en la Ciudad de Huaraz-Ancash. Huaraz: separata de 33 páginas 